\title{
SAPHO Syndrome: Imaging Findings of Vertebral Involvement
}

\author{
(D)A.M. McGauvran, (D)A.L. Kotsenas, (D)F.E. Diehn, (D).T. Wald, (D)C.M. Carr, and (D).M. Morris
}

\begin{abstract}
BACKGROUND AND PURPOSE: Imaging findings in patients with a combination of synovitis, acne, pustulosis, hyperostosis, and osteitis (SAPHO) are often misinterpreted as discitis/osteomyelitis or metastases, resulting in multiple biopsies and delayed diagnosis. We have incidentally noted a semicircular morphology in vertebral body imaging in several cases of SAPHO syndrome with vertebral involvement. Our goal was to evaluate the prevalence of this distinctive morphology in these patients.
\end{abstract}

MATERIALS AND METHODS: A retrospective review of patients with SAPHO syndrome diagnosed between July 1998 and August 2013 was conducted. A descriptive analysis of MR imaging, CT, radiography, bone scanning, and PET imaging was performed for the presence and distribution of vertebral body signal intensity or attenuation changes and/or enhancement; contiguous vertebral body involvement; vertebral body collapse; endplate irregularity; disc space, facet, and spinous process involvement; subligamentous thickening; and paraspinal soft-tissue involvement.

RESULTS: Eighteen patients (16 women [89\%]; mean age, 52.9 years) with SAPHO and spine involvement were included. Contiguous involvement of $\geq 2$ vertebral bodies was found in 16 patients (89\%), with a curvilinear or "semicircular" pattern involving portions of adjacent vertebral bodies in $10(63 \%, P=.14)$. Most intervertebral discs demonstrated absence of abnormal T2 hyperintensity (73\%) and enhancement (89\%). Subligamentous thickening was present in 12 (67\%). Paraspinal soft-tissue involvement was present in 6 (33\%).

CONCLUSIONS: SAPHO syndrome should be included in the differential diagnosis in a patient with a curvilinear or semicircular pattern of vertebral involvement, contiguous vertebral body involvement, and absence of intervertebral disc edema and enhancement.

ABBREVIATION: SAPHO = combination of synovitis, acne, pustulosis, hyperostosis, and osteitis

$\mathrm{T}$ he association of bone disease and chronic cutaneous pustular lesions has been observed since the 1960s, but it was not until 1987 that Chamot et $\mathrm{al}^{1}$ first used the acronym SAPHO to describe this rare group of chronic, relapsing, inflammatory osteoarticular disorders commonly associated with skin manifestations. SAPHO was proposed to refer to a combination of synovitis, acne, pustulosis, hyperostosis, and osteitis as a heading for these syndromes. Cutaneous lesions are characterized by palmoplantar pustulosis, acne conglobata, and/or hidradenitis suppurativa. A wide variety of bone and joint manifestations has been described.

Received October 9, 2015; accepted after revision January 14, 2016.

From the Department of Radiology, Mayo Clinic, Rochester, Minnesota.

Paper previously presented at: Annual Meeting of the American Society of Neuroradiology and the Foundation of the ASNR Symposium, May 17-22, 2014; Montreal, Quebec, Canada.

Please address correspondence to Amy L. Kotsenas, MD, Department of Radiology, Mayo Clinic, 200 First St SW, Rochester, MN 55905; e-mail: Kotsenas.amy@ mayo.edu; @AmyKotsenas

http://dx.doi.org/10.3174/ajnr.A4736
SAPHO syndrome shares some overlapping features with other spondyloarthropathies, including reactive arthritis, psoriatic arthritis, spondyloarthropathy associated with inflammatory bowel disease, and idiopathic ankylosing spondylitis. In children, the disease most commonly presents as a recurrent multifocal osteomyelitis, favoring the long bone metaphysis. This presentation is in contradistinction to that in adults in whom the anterior chest wall, including the sternoclavicular and manubriosternal junctions, is most commonly affected. ${ }^{1-4}$ The spine is also frequently involved in adults. Findings of spinal involvement on conventional radiographic imaging have been reported to include vertebral body osteosclerosis, paravertebral ligament ossification, hyperostosis, and discovertebral junction lesions. Although plain radiographic findings of vertebral involvement have been welldescribed, the radiology literature has only a limited number of case reports and small case series describing imaging findings in detail with other modalities, specifically MR imaging. ${ }^{5-10}$ These advanced imaging findings are often misinterpreted as discitis/ osteomyelitis or metastases. ${ }^{11,12}$ In our experience, this misinter- 


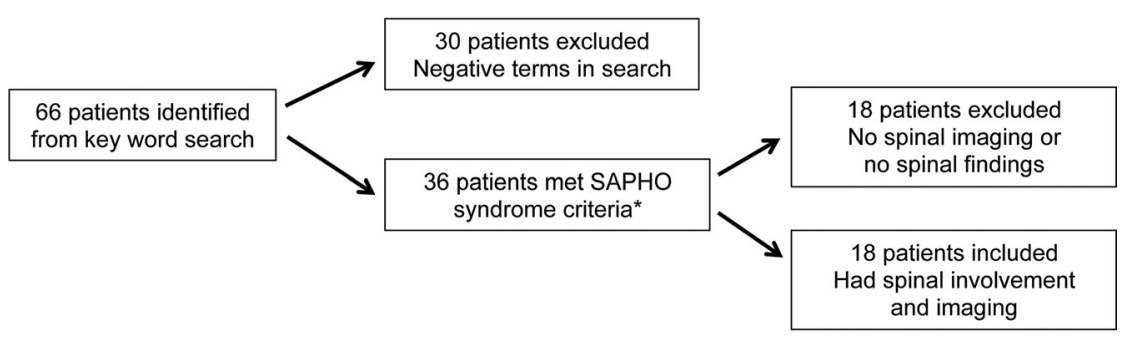

FIG 1. Flow chart demonstrating how patients were narrowed from a large subset to 18 patients with SAPHO syndrome and spinal involvement. *SAPHO criteria were based on Benhamou et al. ${ }^{14}$ ing parameters, and magnet strength varied because many of the scans came from outside facilities. Vertebral body signal intensity was evaluated on T1and T2-weighted MR images and on gadolinium-enhanced T1-weighted images when available. The semicircular pattern of enhancement was recorded if present. The intervertebral discs were evaluated for enhancement, irregularity, associated fluid, and narrowing or wid- pretation can lead to unnecessary biopsies, other invasive procedures, and, ultimately, delayed diagnosis.

Anecdotally, we have commonly observed a "semicircular" pattern of contiguous vertebral body involvement localized to either the anterior or posterior vertebral bodies of the middle segments and adjacent anterior or posterior endplates of the surrounding vertebral bodies. This was first reported by Peffers et al. ${ }^{13}$ The goal of the study was to evaluate the imaging findings in a series of patients with vertebral involvement as part of SAPHO syndrome, with specific attention paid to the prevalence of this unique semicircular morphology in the vertebral bodies.

\section{MATERIALS AND METHODS}

\section{Patients}

Institutional review board approval was obtained, and informed consent was waived for this retrospective review. This study complied with all Health Insurance Portability and Accountability Act requirements. A search of clinical notes and the radiology information management system for patients with the possible diagnosis of SAPHO or any combination of the terms "synovitis," "acne," "pustulosis," "hyperostosis," and "osteitis" between July 1998 and August 2013 identified 66 patients (Fig 1). Of these, 36 patients had a final clinical diagnosis of SAPHO meeting at least 1 of the 4 criteria proposed by Benhamou et $\mathrm{al}^{14}: 1$ ) osteoarticular manifestations of acne conglobata, acne fulminans, or hidradenitis suppurativa; 2) osteoarticular manifestations of palmar plantar pustulosis; 3) hyperostosis involving either the anterior chest wall, spine, or limbs with or without dermatosis; and 4) chronic recurrent multifocal osteomyelitis with or without dermatosis. Eighteen patients were further excluded because they did not have imaging of the spine.

\section{Imaging and Analysis}

We retrospectively reviewed the radiographic imaging findings, including MR imaging, CT, bone scintigraphy, and PET/CT. The images were evaluated by 2 experienced neuroradiologists in consensus (A.L.K. with 15 years, J.M.M. with 7 years). Biopsies, including location, number, and histopathologic and microbiologic culture results, were also recorded.

MR imaging was evaluated for vertebral body signal-intensity changes and/or enhancement; contiguous vertebral body involvement; the presence of vertebral body collapse; endplate irregularity; disc space involvement including enhancement; facet and spinous process involvement; subligamentous thickening; and paraspinal soft-tissue involvement. MR imaging sequences, imag- ening. The number and laterality of the facet joints with signal abnormalities and/or enhancement and the presence of spinous process signal changes were recorded. We looked for ligamentous thickening of the anterior or posterior longitudinal ligaments and ligamenta flava, including thickening that extended to uninvolved vertebral bodies. When there was masslike soft-tissue involvement, the location, maximal diameter (in millimeters), and longitudinal extent of the abnormal tissues were recorded.

CT was evaluated for vertebral body sclerosis or lysis. Radiotracer uptake, if present, was recorded for bone scintigraphy and PET/CT examinations.

\section{Statistical Analysis}

Statistical analysis was performed by using the JMP software package (Version 9.0; SAS Institute, Cary, North Carolina). The Fisher exact test was used to determine the association between the presence of vertebral involvement and sex, the semicircular pattern of involvement, disc space narrowing, and endplate irregularities. Only 2 -tailed tests were used. A $P$ value of $<.05$ was significant.

\section{RESULTS}

\section{Demographics}

Of the 36 patients with SAPHO who were identified (Fig 1), 26 (72\%) were female with an age range of 11-76 years (mean, 44 years; median, 47.5 years). Eighteen patients had spinal imaging available for review, and all demonstrated spinal involvement (50\%; 16 women $[89 \% ; P=.06]$; age range, $23-69$ years).

\section{Vertebral Lesions}

A total of 104 vertebral bodies were involved in 18 patients (Table). One patient had a single level of involvement (6\%), while the remaining 17 patients had a median of 5 vertebral levels involved (range, $2-13$ levels; mean, $5.8 \pm 3.4$ levels). The thoracic spine was most commonly involved ( $n=14,78 \%)$, with $\geq 2$ separate thoracic segments involved in $57 \%$ of patients $(n=8 / 14)$. The lumbosacral spine was the next most commonly involved ( $n=7,39 \%)$, followed by the cervical spine $(n=3,17 \%)$. Multiple regions of the spine were involved in 7 patients (39\%). Contiguous involvement of $\geq 2$ vertebral bodies was found in 16 patients $(89 \%)$, with a curvilinear or semicircular pattern involving the anterior or posterior portions of adjacent vertebral bodies in 10 of these patients $(63 \%$, $P=.14)($ Fig $2 A,-B)$. 
Vertebral body findings per imaging modality

\begin{tabular}{clc}
\hline \multicolumn{1}{c}{ Imaging Modality } & \multicolumn{1}{c}{ Vertebral Body Finding } & No. of Patients \\
\hline MRI $(n=16)$ & T1 hypointensity & 15 \\
T1-weighted images $(n=16)$ & T1 hypointensity $\rightarrow$ T1 hyperintensity & 1 \\
& T2 hyperintensity & 12 \\
T2-weighted images $(n=15)$ & Mixed T2 hypo- and hyperintensity & 3 \\
& Enhancement corresponding to areas of & 9 \\
$\begin{array}{c}\text { Gadolinium-enhanced images } \\
(n=9)\end{array}$ & T1 hypointensity/T2 hyperintensity & 14 \\
CT $(n=14)$ & Sclerosis corresponding to MR signal & abnormalities \\
Technetium TC99m methylene & Increased radiotracer uptake & 10 \\
$\quad \begin{array}{l}\text { diphosphonate 3-phase bone } \\
\text { scan }(n=10)\end{array}$ & & \\
Whole-body FDG-PET/CT $(n=2)$ & No abnormal FDG uptake & 2 \\
\hline
\end{tabular}

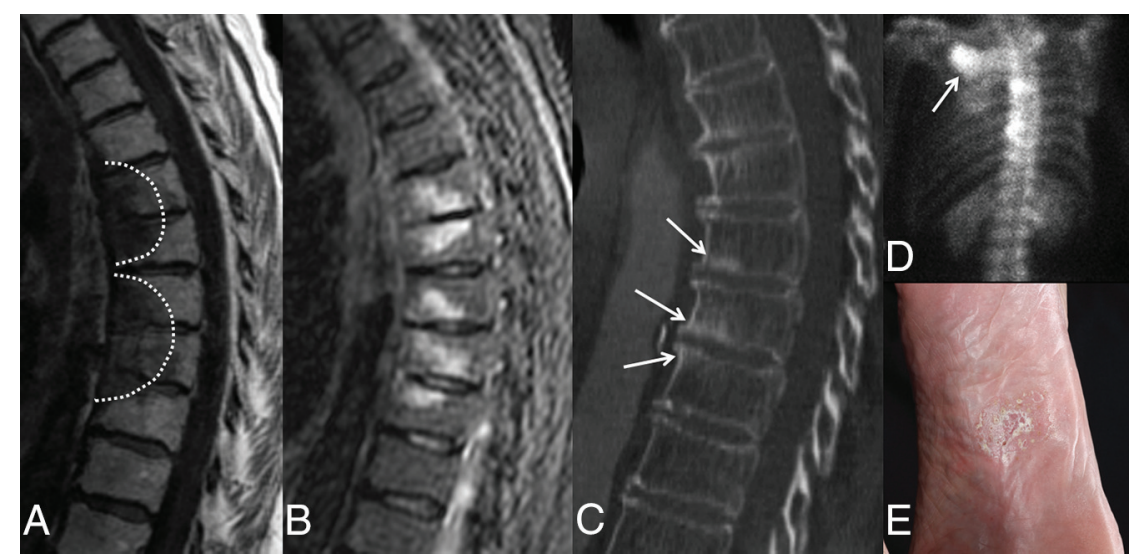

FIG 2. MR imaging, $C T$, and bone scan of the thoracic spine in a 74-year-old woman with back pain. Sagittal T1-weighted $(A)$ and STIR $(B)$ images demonstrate hypo- and hyperintensity, respectively, in a curvilinear or semicircular pattern (dashed line) in contiguous vertebral body segments. Note the absence of abnormal signal within the intervertebral disc spaces. C, Sagittal CT image shows associated sclerosis (arrows) corresponding to levels of abnormal increased signal on MR imaging. $D$, Bone scan, posteroanterior view, demonstrates focal areas of increased radiotracer uptake within thoracic vertebral bodies and the right sternoclavicular joint (arrow). E, Previously undiagnosed plantar pustulosis was evident on physical examination.

MR imaging was available for 16 patients (89\%). Many patients came to our institution with imaging from other facilities. Therefore, there was variation in the MR imaging field strength, sequences, and imaging parameters. T1-weighted images were available in all cases; 1 patient $(6 \%)$ did not have T2-weighted imaging. Vertebral body low-signal intensity on T1-weighted images was seen in 15/16 patients (93\%) with corresponding highsignal intensity on T2-weighted images in 12 patients (75\%), compatible with bone marrow edema (Fig $2 A,-B$ ). One patient $(6 \%)$ had mixed hypo-/hyperintensity on T1-weighted images, while 3 patients (18\%) had mixed hypo-/hyperintensity on T2weighted images, suggestive of concomitant bone marrow edema and cancellous bone sclerosis. Gadolinium-based contrast was administered in 9 patients in whom MR imaging was available (56\%), with $100 \%$ of patients demonstrating enhancement in the same distribution as the signal changes described above. Vertebral body corner erosions were not observed in any patient.

Corresponding noncontrast CT imaging was available in 14 of the 16 patients in whom MR imaging was available. CT demonstrated sclerosis in the areas of MR signal abnormality in $100 \%$ of patients, regardless of the pattern of the MR imaging signal changes (Fig 2C). No lytic lesions were identified.
Nine patients had technetium Tc99m methylene diphosphonate 3-phase wholebody bone scans to correlate with the spinal MR imaging. All 9 patients demonstrated increased radiotracer on MR imaging in the vertebral bodies affected (Fig 2D). There were no discordant areas of vertebral body uptake between the 2 modalities. Extravertebral findings on bone scans are described in further detail below.

In 2 of the $18(11 \%)$ patients with spinal involvement who did not have an MR imaging, the number, level, and contiguity of vertebral lesions were determined solely by nuclear medicine bone scanning in 1 patient and with lumbar spine radiographs in the second. In the first patient, the bone scan demonstrated radiotracer uptake in the noncontiguous T5, T9, T11, and L1 vertebral bodies and right sacroiliac joint. In the second, lumbar spine radiographs demonstrated attenuated vertebral body sclerosis from L3 to the sacrum and partial fusion of the left sacroiliac joint.

Thirteen of 18 patients (72\%) had plain radiographs. One patient had normal findings on plain radiographic imaging (8\%). Twelve patients had vertebral body sclerotic changes, including shiny corners (92\%). Despite abnormal findings on MR imaging, no abnormal FDG uptake was seen in the 2 patients with whole-body FDG-PET/CT.

\section{Facet Joints/Spinous Processes}

There were 34 facet joints involved with $\mathrm{T} 2$ hyperintensity and/or enhancement in 7 patients (39\%), with unilateral involvement at 6 levels (3 patients, 17\%) and bilateral involvement at 14 levels (4 patients, 22\%). Spinous process T2 hyperintensity or enhancement or both was observed at 15 levels in 5 patients (28\%), all with concurrent uni- or bilateral facet joint involvement at the same levels (Fig 3).

\section{Disc Spaces}

MR imaging demonstrated at least 1 intervening or adjacent disc space that was narrowed in 8 of $16(50 \%)$ patients, with irregular endplates observed in 7 patients $(43 \%)$. The combination of disc space narrowing with endplate irregularity was noted in 5 patients who had MR imaging $(62 \% ; P=.11)$. High signal on T2-weighted images in the disc was seen in 5 patients (31\%). Disc space enhancement on gadolinium-enhanced T1-weighted images was observed in 2 of 9 patients receiving gadolinium (22\%). The combination of disc space narrowing, abnormal T2 hyperintensity, and endplate irregularity was observed in 3 patients (18\%), while only 1 patient (11\%) had all 4 findings: disc space narrowing, abnormal T2 hyperintensity, endplate irregularity, and disc space enhancement.

AJNR Am J Neuroradiol 37:1567-72 Aug 2016 www.ajnr.org 


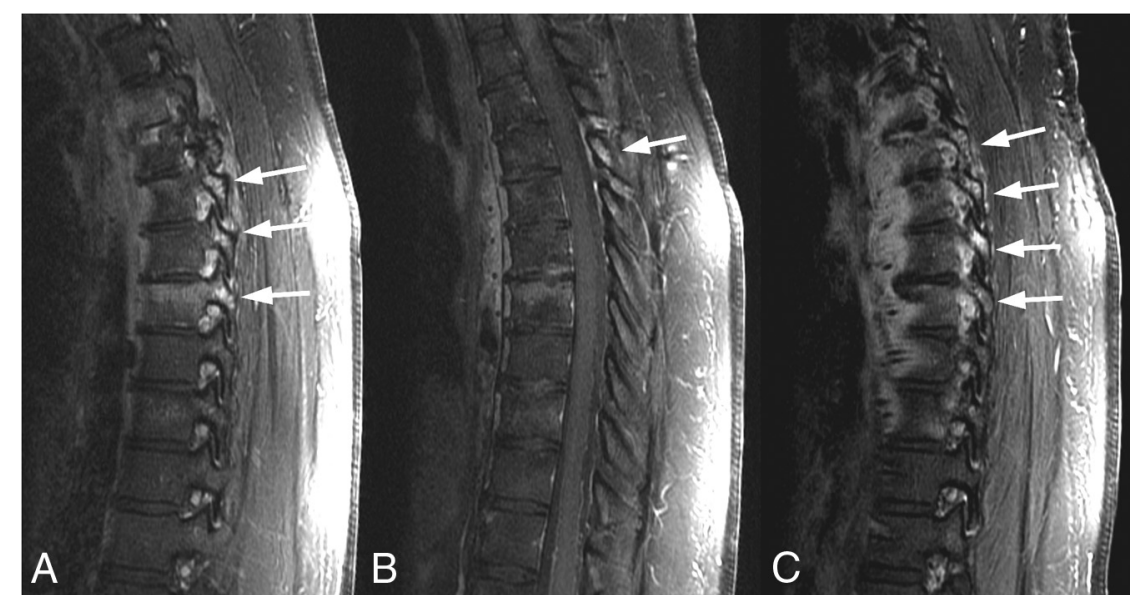

FIG 3. MR images in a 32-year-old woman with back pain. Sagittal T1-weighted fat-saturated images of the thoracic spine following administration of gadolinium demonstrate multilevel enhancement (arrows) of the spinous processes (B) and bilateral facet joints ( $A$ and $C$ ).

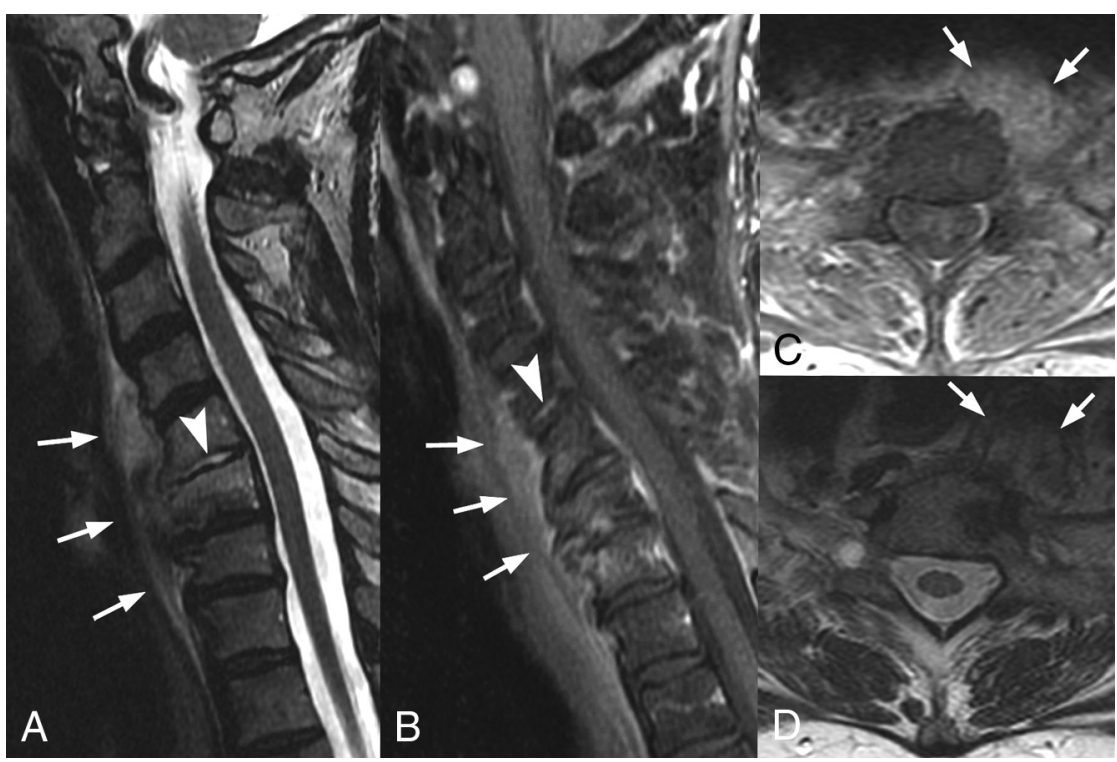

FIG 4. MR images in a 69-year-old woman with cervical and thoracic back pain. A, Sagittal T2weighted fat-suppressed image of the cervical spine demonstrates paravertebral soft-tissue thickening (arrows), extending from the C3-C4 interspace to T3. Note the abnormally high T2-weighted signal within the $\mathrm{C} 5-\mathrm{C} 6$ disc (arrowhead). B. Sagittal T1-weighted gadolinium-enhanced fat-suppressed image demonstrates corresponding enhancement of the paravertebral soft tissue (arrows) with disc space enhancement (arrowhead). C, Axial T1-weighted gadoliniumenhanced fat-suppressed image demonstrates enhancement of the thickened paravertebral soft tissues (arrows). D, Axial T2-weighted fat-suppressed image further demonstrates the extent of the paravertebral soft-tissue thickening (arrows).

\section{Soft Tissues}

Thickening of the anterior longitudinal ligament was present in 12 patients $(80 \%)$ and extended to otherwise uninvolved vertebral bodies in 3 patients (25\%). No other spinal ligaments were involved. Paraspinal soft-tissue involvement consisted of masslike soft-tissue thickening with enhancement (Fig 4). This was observed in 6 patients (37\%), with a range of 1-6 vertebral levels involved. Masslike paraspinal soft-tissue involvement did not exceed $15 \mathrm{~mm}$ in maximal diameter in any case (range, 2-15 mm).

\section{Extraspinal Involvement}

Seventeen of 18 patients (94\%) had typical skin manifestations. Ten (59\%) of the 17 patients had palmoplantar pustulo- sis (Fig 2E), 2 patients (12\%) had acne conglobata, 1 patient $(6 \%)$ had hidradenitis suppurativa, and 6 patients (35\%) had nonspecific dermatoses. Two patients $(12 \%)$ had $>1$ skin manifestation, with 1 patient having both palmoplantar pustulosis and acne conglobata, while the other patient had both palmoplantar pustulosis and hidradenitis suppurativa. Seven of the 18 patients (39\%) with SAPHO syndrome and spinal involvement also had concurrent sternoclavicular involvement (Fig 2), while 4 patients (22\%) had involvement of the first costovertebral joint. Four of the 18 patients $(22 \%)$ had plain radiographs of the sacroiliac joints, with 1 patient $(25 \%)$ demonstrating sclerosis and the remaining 3 patients demonstrating no involvement of the sacroiliac joint.

\section{Pathology/Microbiology}

Twelve patients (67\%) underwent biopsy; 7 patients had a single biopsy, 3 patients had 2 biopsies, and 2 patients had 3 biopsies, totaling 19 biopsies. The vertebral body was the most commonly biopsied location $(n=11)$, followed by the paravertebral soft tissues $(n=5)$ and disc interspace $(n=1)$. In 2 patients with spinal involvement, a concomitant sternal lesion was biopsied. Pathology was negative for malignancy in all biopsy specimens $(n=19)$, including 2 patients (11\%) with a known history of malignancy. Microbiologic cultures were also negative for infection in all 8 patients who had microbiologic testing performed.

\section{Inflammatory/Infectious Markers}

C-reactive protein levels were available in 13 of 18 patients $(72 \%)$, with elevated levels in 8 patients $(62 \%)$. Sedimentation rate levels were available in 16 of 18 patients (89\%), with elevated levels in 6 patients $(38 \%)$. White blood cell counts were available in 17 of 18 patients (94\%), with all patients having normal levels.

\section{DISCUSSION}

A curvilinear or a semicircular pattern of contiguous vertebral body involvement localized to either the anterior or posterior vertebral bodies of the middle segments and adjacent anterior or posterior endplates of the surrounding vertebral bodies was found in most of our patients (63\%) with SAPHO and spinal involvement. The high prevalence of this semicircular pattern of vertebral body signal alteration and enhancement may help to 
differentiate SAPHO syndrome from metastases, which tend to be randomly distributed throughout the spine. When combined with a relatively low prevalence of abnormal disc space T2-signal and enhancement, the high prevalence of the semicircular pattern may also be helpful to radiologists in cases in which spinal infection is being considered. Correlation with CT may be beneficial because sclerosis in the areas of MR signal abnormality was present in $100 \%$ of our patients, regardless of the pattern of the MR signal changes. Recognition of these findings by radiologists should prompt clinical consultation with dermatology and/or rheumatology to assess typical skin and other musculoskeletal manifestations.

Osteosclerosis of the vertebral bodies in SAPHO syndrome has been described by Leone et $\mathrm{al}^{15}$ and may progress to produce diffuse and generalized sclerosis with development of hyperostosis in more chronic cases. Prior case reports ${ }^{16-18}$ have shown the utility of $\left[{ }^{18} \mathrm{~F}\right]$ FDG-PET/CT to differentiate active from healed chronic inflammatory lesions because PET/CT shows increased uptake only in lesions with active inflammation. The chronic osteosclerotic changes seen in our patients are hypothesized to represent a quiescent phase of the disease, which likely accounts for the normal FDG uptake in the 2 patients who had PET/CT scans in our series.

In contrast to a study performed by Laredo et al, ${ }^{6}$ our patients were more likely to have involvement at contiguous levels. In addition, while our patients had frequent involvement of vertebral body corners, we did not identify erosion in any case. Because we are a tertiary referral center, our patients may have presented at a later clinical stage. In fact, nearly all our patients presented after other manifestations such as skin lesions or sternoclavicular involvement, both of which are considered highly specific, were evident.

The frequency of spondylodiscitis in SAPHO syndrome has been reported to be $9 \%-32 \% .{ }^{10}$ Prior studies have shown chronic sterile nonspecific inflammation from intervertebral disc biopsies in patients with SAPHO syndrome, equivalent to the Andersson lesion, which has been identified in $4.5 \%$ of patients with ankylosing spondylitis. ${ }^{5,10}$ Others have suggested that spondylodiscitis is due to Propionibacterium acnes. ${ }^{19}$ Although disc space involvement with fluidlike signal on T2-weighted images and disc space enhancement have been previously described in patients with SAPHO syndrome, ${ }^{5,7}$ our study showed that most intervertebral discs in patients with SAPHO syndrome demonstrated the absence of both fluidlike signal on T2-weighted images (69\%) and disc space enhancement (78\%). The absence of these findings may be helpful in distinguishing SAPHO involvement from spinal infection.

We found single-level vertebral involvement rare, with contiguous involvement of $\geq 2$ levels in nearly $90 \%$, often with $\geq 5$ levels involved. This finding is in contrast to cases of spondyloarthropathy, in which it is uncommon to see contiguous involvement. ${ }^{6}$

Nachtigal et $\mathrm{al}^{7}$ previously described the paravertebral soft tissues in patients with SAPHO involvement of the spine possibly showing abnormal signal intensity. We found similar results, with thickening and enhancement of the anterior longitudinal ligament present in $37 \%$ of patients with SAPHO syndrome. These findings further complicate the differential diagnosis of SAPHO syndrome versus infection but may help to differentiate it from spondyloarthropathies, which, to our knowledge, have only been reported to affect the supraspinous ligament, interspinal ligaments, and ligamenta flava. ${ }^{20}$ Additionally, in the 2 patients with vertebral body involvement determined solely on nuclear medicine bone scans or plain radiographs, both demonstrated unilateral sacroiliac joint involvement. Depasquale et $\mathrm{al}^{11}$ and Leone et al ${ }^{15}$ have both described increased sclerosis and hyperostosis, particularly at the iliac side of the joint, in patients with SAPHO. When seen in patients with features of moderate sacroiliitis, this presentation was thought to be highly suggestive of SAPHO syndrome and helped to differentiate it from other spondyloarthropathies.

Peffers et $\mathrm{al}^{13}$ first described a subset of patients with nonbacterial osteitis involving the thoracic spine. Spinal involvement in our study had a strong female predominance and preferentially affected the thoracic spine. This is in contrast to osteomyelitis and discitis, in which males are affected twice as often as females and the lumbar spine is most commonly affected. ${ }^{21}$

MR imaging of the spine and sacroiliac joints has played a key role in patients with spondyloarthropathies and has led to improved understanding of the course of the disease and to an earlier diagnosis. ${ }^{20}$ We suggest an imaging protocol consisting of at least a sagittal T1-weighted turbo spin-echo sequence, sagittal fat-saturated T2-weighted turbo spin-echo sequence or STIR sequence with high resolution, and a sagittal fat-saturated gadolinium-enhanced T1-weighted sequence for evaluation of patients with suspected SAPHO syndrome, spondyloarthropathy, or osteomyelitis/discitis.

Our study had a number of limitations. This was a retrospective review without a control group, and images were reviewed in consensus rather than independently by the 2 neuroradiologists, which might limit the reproducibility. Additionally, the number of patients was small; however, SAPHO syndrome is an uncommon entity, and ours is one of the largest studies to date. The MR imaging protocols were not standardized. For example, not every patient had a fat-saturated sequence, 1 patient lacked a T2weighted sequence, and 7 of 16 patients (44\%) did not receive intravenous contrast material. Variation in MR imaging field strength and imaging protocols was a relatively minor limitation because signal change was noted on precontrast T1-weighted images in all patients. Finally, biopsy and microbiologic results were not available in half of the patients, perhaps due to the presence of other highly specific anterior chest wall and/or skin manifestations.

Additional studies involving larger numbers of patients with SAPHO syndrome and comparison groups of other infectious and noninfectious spinal inflammatory conditions are needed to 
determine whether this semicircular pattern is a unique spinal finding in patients with SAPHO syndrome.

\section{CONCLUSIONS}

In a patient with a curvilinear or semicircular pattern of contiguous vertebral involvement, sclerosis along ligamentous attachment sites, and the absence of intervertebral disc abnormal T2 hyperintensity and enhancement, SAPHO syndrome should be included in the differential diagnosis and clinical consultation with dermatology and/or rheumatology should be considered. This may prevent the imaging findings from being misinterpreted as discitis/osteomyelitis or metastases, with subsequent potential reduction in the number of unnecessary biopsies and delayed diagnoses.

\section{ACKNOWLEDGMENTS}

We thank Sonia Watson, $\mathrm{PhD}$, for her assistance in editing the manuscript.

\section{REFERENCES}

1. Chamot AM, Benhamou CL, Kahn MF, et al. Acne-pustulosis-hyperostosis-osteitis syndrome: results of a national survey-85 cases [in French]. Rev Rhum Mal Osteoartic 1987;54:187-96 Medline

2. Boutin RD, Resnick D. The SAPHO syndrome: an evolving concept for unifying several idiopathic disorders of bone and skin. AJR AmJ Roentgenol 1998;170:585-91 CrossRef Medline

3. Kahn MF, Chamot AM. SAPHO syndrome. Rheum Dis Clin North Am 1992;18:225-46 Medline

4. Maugars Y, Berthelot JM, Ducloux JM, et al. SAPHO syndrome: a followup study of 19 cases with special emphasis on enthesis involvement. J Rheumatol 1995;22:2135-41 Medline

5. Akisue T, Yamamoto T, Marui T, et al. Lumbar spondylodiscitis in SAPHO syndrome: multimodality imaging findings. J Rheumatol 2002;29:1100-01 Medline

6. Laredo JD, Vuillemin-Bodaghi V, Boutry N, et al. SAPHO syndrome: MR appearance of vertebral involvement. Radiology 2007;242: 825-31 CrossRef Medline

7. Nachtigal A, Cardinal E, Bureau NJ, et al. Vertebral involvement in SAPHO syndrome: MRI findings. Skeletal Radiol 1999;28:163-68 CrossRef Medline

8. Perez C, Hidalgo A, Olier J, et al. MR imaging of multifocal spondy- lodiskitis as the initial manifestations of SAPHO syndrome. AJR Am J Roentgenol 1998;171:1431-32 Medline

9. Tohme-Noun C, Feydy A, Belmatoug N, et al. Cervical involvement in SAPHO syndrome: imaging findings with a 10-year follow-up. Skeletal Radiol 2003;32:103-06 CrossRef Medline

10. Toussirot E, Dupond JL, Wendling D. Spondylodiscitis in SAPHO syndrome: a series of eight cases. Ann Rheum Dis 1997;56:52-58 CrossRef Medline

11. Depasquale R, Kumar N, Lalam RK, et al. SAPHO: what radiologists should know. Clin Radiol 2012;67:195-206 CrossRef Medline

12. Hong SH, Choi JY, Lee JW, et al. MR imaging assessment of the spine: infection or an imitation? Radiographics 2009;29:599-612 CrossRef Medline

13. Peffers G, James SL, Stirling A, et al. Thoracic spine osteitis: a distinct clinical entity, a variant of SAPHO or late-onset non-bacterial osteitis? Rheumatology (Oxford) 2012;51:191-93 CrossRef Medline

14. Benhamou CL, Chamot AM, Kahn MF. Synovitis-acne-pustulosis hyperostosis-osteomyelitis syndrome (SAPHO): a new syndrome among the spondyloarthropathies? Clin Exp Rheumatol 1988;6: 109-12 Medline

15. Leone A, Cassar-Pullicino VN, Casale R, et al. The SAPHO syndrome revisited with an emphasis on spinal manifestations. Skeletal Radiol 2015;44:9-24 CrossRef Medline

16. Inoue $\mathrm{K}$, Yamaguchi $\mathrm{T}$, Ozawa $\mathrm{H}$, et al. Diagnosing active inflammation in the SAPHO syndrome using 18FDG-PET/CT in suspected metastatic vertebral bone tumors. Ann Nucl Med 2007;21:477-80 CrossRef Medline

17. Patel CN, Smith JT, Rankine JJ, et al. F-18 FDG PET/CT can help differentiate SAPHO syndrome from suspected metastatic bone disease. Clin Nucl Med 2009;34:254-57 CrossRef Medline

18. Takeuchi K, Matsusita M, Takagishi K. A case of SAPHO (synovitisacne-pustulosis-hyperostosis-osteomyelitis) syndrome in which $[18 \mathrm{~F}]$ fluorodeoxyglucose positron emission tomography was useful for differentiating from multiple metastatic bone tumors. Mod Rheumatol 2007;17:67-71 CrossRef Medline

19. Gerster JC, Lagier R, Livio JJ. Propionibacterium acnes in a spondylitis with palmoplantar pustulosis. Ann Rheum Dis 1990;49:337-38 Medline

20. Sieper J, Rudwaleit M, Baraliakos X, et al. The Assessment of SpondyloArthritis international Society (ASAS) handbook: a guide to assess spondyloarthritis. Ann Rheum Dis 2009;68(suppl 2):ii1-44 Medline

21. Chen WH, Jiang LS, Dai LY. Surgical treatment of pyogenic vertebral osteomyelitis with spinal instrumentation. Eur Spine J 2007;16: 1307-16 CrossRef Medline 